\title{
EL FINANCIAMIENTO: PRINCIPAL RETO DEL MICROEMPRENDIMIENTO EN ECUADOR
}

\section{FINANCING: MAIN CHALLENGE OF MICRO-ENTREPRENEURSHIP IN ECUADOR}

Emma Yolanda Mendoza Vargas, Mgs. Magíster en Dirección de Empresas con énfasis en Gerencia Estratégica (Ecuador). Docente la Unidad de Estudios a Distancia de la Universidad Técnica Estatal de Quevedo, Ecuador. https://orcid.org/0000-0002-0220-4328 emendoza@uteq.edu.ec

Harold Elbert Escobar Terán, Mgs. Magíster Universitario en Ingeniería de Sistemas de Decisión (Ecuador). Docente de la Universidad Técnica Estatal de Quevedo, Ecuador. https://orcid.org/0000-0001-9165-6627 hescobar@uteq.edu.ec

Jhon Alejandro Boza Valle, Ph.D.

Doctor en Ciencias Económicas (Cuba). Docente Titular de la Facultad de Ciencias Empresariales de la Universidad Técnica Estatal de Quevedo, Ecuador. http://orcid.org/0000-0002-3458-3844

jboza@uteq.edu.ec

\section{ARTÍCULO DE INVESTIGACIÓN}

Recibido: 4 de septiembre de 2019.

Aceptado: 31 de octubre de 2019.

\section{RESUMEN}

Detrás de la dinámica de la estructura macroempresarial se encuentra la gestión del microemprendimiento, que hoy constituye una de las principales fuentes de empleo en la

ISSN: 1390-9320, Vol. 6, No. 6, diciembre 2019 
nación ecuatoriana, y específicamente en el territorio de Quevedo. Sin embargo, las fallas en el manejo financiero constituyen una de las principales razones por las cuales muchos negocios fracasan o no alcanzan altos índices de crecimiento; de ahí la pertinencia de explicar por qué el financiamiento constituye uno de los criterios claves a tener en cuenta a la hora de iniciar algún negocio, y lograr y mantener el éxito. Para desarrollar la investigación descriptiva se partió de un enfoque cuantitativo con el empleo de la revisión bibliográfica documental; así como de la encuesta. Se deduce que existe una escasa información acerca de las ventajas que implica un adecuado diseño financiero de cada una de las actividades que se desarrollan dentro de la microempresa, para tomar decisiones a corto, mediano y largo plazo. Por otra parte, se desconoce cuáles son las entidades financieras a las cuales resulta más conveniente acudir, así como las facilidades que pueden proporcionar en apoyo a este tipo de iniciativas a menor escala de la economía.

Palabras Clave: microemprendimiento, financiamiento, emprendedor, mercado, economía

\section{ABSTRACT}

Behind the dynamics of the macro-business structure is the management of microentrepreneurship, which today constitutes one of the main sources of employment in the Ecuadorian nation, and specifically in the territory of Quevedo. However, failures in financial management are one of the main reasons why many businesses fail or do not reach high growth rates; hence the relevance of explaining why financing is one of the key criteria to consider when starting a business, and achieve and maintain success. To develop descriptive research, a quantitative approach was taken with the use of documentary literature review; as well as the survey. It follows that there is little information about the advantages of an adequate financial design of each of the activities carried out within the microenterprise, to make decisions in the short, medium and long term. On the other hand, it is unknown which are the financial institutions to which it is more convenient to go, as well as the facilities that they can provide in support of this type of initiatives on a smaller scale of the economy.

Keywords: micro entrepreneurship, financing, entrepreneur, market, economy.

ISSN: 1390-9320, Vol. 6, No. 6, diciembre 2019 


\section{INTRODUCCIÓN}

El microemprendimiento constituye uno de los fenómenos que hoy más movilizan a la economía de América Latina. Según una investigación realizada por Torres (2006) en más de una decena de países de la región, el $94.6 \%$ de las organizaciones empresariales se encontraban para ese entonces dentro de clasificación micro, lo cual ocupaba una cifra mayor al $40 \%$ de la fuerza laboral activa. Ello significa que el sector constituye un paliativo para el problema del desempleo en los países subdesarrollados, teniendo en cuenta que pocos Estados de esta territorialidad implementan una política de empleo que satisfaga la demanda de trabajo de su población.

A pesar de los esfuerzos de los emprendedores, no se puede ocultar que un alto índice de estos esfuerzos cierran en los primeros años de funcionamiento, son muy pocos los que perduran y llegan a tener características generacionales, sin embargo a lo señalado, los emprendedores relucen sus características de resiliencia por cuanto a pesar del fracaso de una idea, inmediatamente están embarcándose en otra, asumiendo riegos y en ocasiones embistiendo casi a ciegas, lo que desnuda la poca incidencia de los gobiernos locales en el desarrollo del emprendimiento y la necesidad de que la academia se vincule aún más con los emprendedores en procura de lograr inversiones que aseguren éxito empresarial y sostenibilidad (Escobar, 2018).

Ecuador constituye uno de los países latinoamericanos en los cuales durante los últimos años se aprecia la aparición de múltiples pequeñas, medianas y microempresas, sobre todo concentrados en el sector del comercio (53\%) y los servicios (39\%) (EKOSNEGOCIOS, 2015). Sin embargo, no porque existan, esas organizaciones empresariales tienen garantizada su productividad, entendida como el nivel de eficiencia alcanzada en correspondencia con las metas propuestas. La baja productividad del sector hoy constituye uno de los principales objetos de estudio de especialistas de organismos económicos nacionales e internacionales como la CEPAL (2013).

Mungaray y Ramírez (2007) atribuye este bajo impacto de los microemprendimientos a la escasa capacitación con que los empleadores y empleados se someten a invertir en el

ISSN: 1390-9320, Vol. 6, No. 6, diciembre 2019 
sector, otras de las razones radica en el carácter informal con que se emprenden estos proyectos y que lo pone en desventaja dentro del mercado en la medida que no tienen acceso a determinadas facilidades de las cuales sí goza el sector formal, en materia de seguridad social, inversión, reconocimiento, financiamiento (CEPAL, 2013); y es precisamente este último aspecto, uno de los que más desfavorece la posibilidad de éxito de las microempresas.

El estudio desarrollado por la Universidad Andina Simón Bolívar y el Young Américas Business Trust (YABT), "Análisis de la situación actual del emprendimiento: sus actores públicos y privados" (EKOSNEGOCIOS, 2015), revela que apenas el 37\% de proyectos incluyen acciones dirigidas a evaluar la oferta de fuentes de financiamiento especializadas en la entrega de recursos para el emprendimiento. A ello súmesele que la mayoría de los microemprendimientos utilizan fuentes no formales de crédito y de préstamos, y al no acudir a las fuentes formales para el financiamiento de sus inversiones en el comienzo y el proceso de desempeños las expectativas para aumentar el dinamismo de ese sector suelen ser bajas.

Las actividades que se insertan dentro del microempredimiento, por muy corto alcance que pretendan, requiere de una adecuada administración para aprovechar las oportunidades, atreverse a los giros de productos y tomar riesgos dentro de los mercados; sin un financiamiento estable, las posibilidades de aplicar estas estrategias resultas escasas, por tanto, constituyen las estructuras económicas que más vulnerables se encuentran dentro de la organización económica del país, debido a la recurrente desaceleración o recesión de la economía.

El fracaso resulta un fantasma para del desempeño de los emprendedores de ese sector, en tanto operan sin políticas públicas o económicas que ofrezcan facilidades para su desenvolvimiento dentro del mercado, y ello inciden desfavorablemente en otros aspectos como realización personal de los trabajadores y la supervivencia de sus familias. Debido al dinamismo que hoy genera en la economía este tipo de proyectos empresariales, resulta pertinente explicar por qué el financiamiento constituye uno de los criterios claves a tener en cuenta a la hora de iniciar algún negocio, y lograr y mantener el éxito.

ISSN: 1390-9320, Vol. 6, No. 6, diciembre 2019 


\section{REVISIÓN TEÓRICA}

\section{Microempresas y emprendedores}

No existe un acuerdo en torno a la conceptualización de la microempresa. Según explica Domínguez (2005), depende de la perspectiva por la cual se mire, algunos alegan que cuando tienen un número menor a 10 empleados puede concebirse como este tipo de negocio, o cuando sus bienes comprenden menos de $\$ 5,000$ en activos fijos o menos de dos veces el producto nacional bruto per cápita en edificios y equipo); o cuando sus ventas resultan menores de $\$ 500$ por mes en ventas totales o mensualmente menos de tres veces el salario mensual mínimo); o cuando circulan dentro del sector informal, no autorizado, o no regulado) o la estructura gerencial.

Otros indicadores que algunos autores evalúan, es el mercado principal en el cual se desempeñan, o si se insertan dentro del sector de las pequeñas propiedades en el área rural del país. Asimismo, los bancos, donantes, y empresarios que pertenecen al sector formal también construyen sus propias definiciones acerca del asunto, pues de ello depende el interés en invertir en los micronegocios, o no.

Nos obstante, existen otros elementos que por lo general caracterizan a los microemprendimientos, se tratan casi siempre de negocios familiares, que implican pequeños activos, empleados y volúmenes de ventas. Otro de las peculiaridades de esas organizaciones es que son operadas por personas que viven en la pobreza, con un bajo nivel educativo, y por consiguiente, con una escasa capacitación en materia empresarial. No se encuentran preparados para invertir y mucho menos emplear tecnologías sofisticadas, de manera que regularmente intervienen en el sector bajo del comercio y su posibilidad de producción se encuentra bien limitada.

Asociado a este tipo de empresa se encuentran los emprendedores, una terminología que a pesar de no ser novedosa, en la actualidad adquiere un mayor reconocimiento por los estudios relacionados con los negocios y la economía de los países.

Una definición que se ajusta a los objetivos de este artículo la ofrece Schumpeter (2012), desde inicios del siglo XX; al referirse a esta figura, la concibe como un ente reformador del mecanismo de producción dentro del mercado, en tanto se trata de una persona que

ISSN: 1390-9320, Vol. 6, No. 6, diciembre 2019 
sustenta su proyecto empresarial en la invención y la innovación, llegando, con pocos recursos, a posicionar su producto dentro de la competencia (Drucker, 1985), independientemente de cuan nuevo o viejo se encuentre.

La labor del emprendedor se ve impulsada además por sus dotes creativas, que le permiten aprovechar las oportunidades y correr riesgos dentro del mercado. Un autor más contemporáneo, Kirzner (1998)) lo postula entre los agentes que pueden tomar buenas decisiones pues comprenden una visión que les permite adelantarse a los errores del mercado para colocar el producto o servicio entre las mejores ofertas.

Por su parte, Borja (2015), basa los logros del emprendedor en su intuición, y en las características de su personalidad como la autoestima, la confianza en sus capacidades, disciplina en la toma de decisiones, espíritu de triunfador, así como las habilidades que presenta como líder para poder organizar el trabajo y que las personas le correspondan a sus exigencias y demandas.

Sin embargo, el negocio del emprendimiento empresarial no basa su éxito exclusivamente en las características personales de los individuos, uno de los aspectos que más influye en el rendimiento de una organización de este tipo es el financiero, que en la actualidad representa uno de las dificultades más importantes para el pequeño empresario.

De manera que cuando se percibe al microemprendedor como agente de cambio y desarrollo económico y social (Schumpeter, 2012), tiene que evaluarse también el tipo de apoyo que sustenta su negocio, y para el cual no están creadas las condiciones, a nivel estatal ni empresarial. De manera que las instituciones del Estado y los negocios más fructíferos pocas veces aportan una ayuda para las microempresas, en aspectos como las fuentes de abasto, financiamiento, ventaja en las negociaciones y en las inversiones.

\section{Prácticas para el financiamiento de las microempresas}

El financiamiento constituye una de las principales herramientas de las que se vale cualquier tipo de empresa para optimizar los recursos. De acuerdo con Quiñonez (2013), determinar qué clase de financiamiento emplear en determinada organización, ya sea a corto o lejano plazo, va a depender de las características que tiene esa entidad. Domínguez (2005) ha definido este proceso como la adquisición de recursos o formas de pago, que se

ISSN: 1390-9320, Vol. 6, No. 6, diciembre 2019 
emplean para comprar los bienes de capital que requiere la empresa, ya sea para iniciar su gestión o dar continuidad a esta.

Se habla de financiamiento interno cuando el capital se deriva y se invierte en reservas, previsiones, adquisición de mayor capital; mientras que la financiación externa se debe al dinero que no pertenece a la empresa propiamente dicha y que por lo general se dispone para gastos más excesivos que los anteriormente mencionados, o cuando la organización no se encuentra en condiciones de adquirir dinero de manera autónoma.

El estudio desarrollado por la USAID (2005) en Ecuador reveló cuáles son las explicaciones de que muchas empresas fracasen por motivo de la gestión del financiamiento. Entre ellos, que aproximadamente el $15 \%$ de los emprendedores consultados creían en que ellos podían asumir tanto el inicio como las emergencias del negocio con sus fuentes personales, que casi siempre afloran de las cuentas de ahorro.

Por otro lado, cuando no cuentan con un patrimonio efectivo para el financiamiento, recurren a fuentes informales de crédito, como son las familias, amigos, anticipos de los clientes y proveedores. Solo el $11 \%$ refirió pedir capital en instituciones formales. Este fenómeno responde también a las políticas de préstamos que tradicionalmente se ha establecido en Ecuador, las cuales, en su mayoría, no confían en los nuevos negocios. La mayoría de los prestamistas que pudiesen considerar los microemprendedores exigirán que la empresa haya estado funcionando durante al menos un año, de manera que puedan estas, de alguna manera garantizar que el dinero regresará a sus fondos.

Esta situación ayuda a comprender porqué casi todos los microemprendimientos basan la financiación de los procesos en dos fuentes: ventas y ahorros (USAID-Ecuador, 2005); recurren a fuentes informales en lugar de acudir a los bancos, compañías financieras, cooperativas de ahorro y crédito, cooperativas, y organizaciones no gubernamentales. Pocas personas confían en el establecimiento de un vínculo con un centro financiero, pues no lo tienen estipulado como una estrategia comercial para garantizar sus recursos, y por demás sus ganancias.

Se aprecia el conservadurismo que persiste en el país, en tanto los emprendedores interpretan la diversificación de las fuentes de financiamiento con el endeudamiento, lo cual

ISSN: 1390-9320, Vol. 6, No. 6, diciembre 2019 
conlleva a un problema mayúsculo, cuando el negocio depende de las ganancias internas, la posibilidad de crecer se vuelven casi nulas, sobre todo por la baja productividad y los limitados niveles de ingreso y rentabilidad que generalmente presentan este tipo de entidades (Kirzner, 1998). En cambio, una gestión financiera acorde con las necesidades de corto y largo plazo de las microempresas suelen significar un mecanismo beneficioso para incrementar las posibilidades de los negocios en el terreno de cualquier mercado.

\section{Presupuestos metodológicos}

Explicar por qué el financiamiento constituye uno de los criterios claves a tener en cuenta a la hora de iniciar algún negocio, y lograr y mantener el éxito, al ser una temática escasamente tratada en el territorio de Quevedo, requiere de la formulación de una investigación de tipo descriptiva con enfoque cuantitativo. Ello permitirá llegar a la mayor cantidad de información a partir de los datos que generan los propios microempresarios, en el menor tiempo posible.

Para conocer los aspectos relacionados con el financiamiento que sustentan o no a las microempresas, se requirió primeramente de la implementación del método Bibliográfico Documental, que propició profundizar en temas como las fuentes del financiamiento, a qué se dirigen las inversiones, los errores que se cometen en el manejo de la economía de esos locales y cuáles son las estrategias que emplean los microempresarios para que el proyecto funcione y resulte en ganancias.

Se emplea, además, el Método Inductivo-Deductivo que supone el contraste de los datos devenidos de la aplicación de las técnicas de investigación con las concepciones teóricas, de manera que se pueda arribar a conclusiones con un carácter de mayor generalidad, así como explicar aspectos particulares del fenómeno que aquí se estudia.

La encuesta constituye la principal técnica utilizada, mediante la aplicación de un cuestionario que interroga acerca de los tipos de locales que predominan bajo el modelo del Microemprendimiento, las razones para iniciar los negocios que entran bajo esta categoría del trabajo por cuenta propia, las particularidades e irregularidades que presenta el proceso de financiamiento; todo lo cual ayudan a describir en términos económicos cómo se mantienen esas pequeñas organizaciones empresariales.

ISSN: 1390-9320, Vol. 6, No. 6, diciembre 2019 
Como todos los microempresarios de la región de Quevedo tenían las mismas condiciones para convertirse en el objeto de estudio, y constituyen una población demasiado mayoritaria, se recurrió a la realización de un muestreo intencionado, donde, quienes participaran en la encuesta fueran personas con más de un año de experiencia en el sector, se eligió a una representación de cada género, y que se desarrollaran en negocios de comercio, pues son los locales que más proliferan en el territorio y por tanto son personas a las cuales resulta posible acceder. En total se aplicó el cuestionario 320 sujetos.

\section{ANÁLISIS DE LOS RESULTADOS}

Emprender un negocio, en la mayoría de los casos, requiere de una importante inversión capital para impulsar lo que sería la primera parte del emprendimiento: adquirir un local adecuado, capacitar al personal o contratar a trabajadores ya capacitados, invertir en materia prima, realizar el marketing de los productos o servicios. A su vez, este primer financiamiento necesita ser administrado de forma eficaz de manera que la primera inversión resulte más importante que las ganancias obtenidas en ese primer periodo para evitar adeudos, lo cual requiere de habilidades como la astucia, la toma de decisiones, asumir riesgos por parte del emprendedor.

El financiamiento de este tipo de negocio en Ecuador, generalmente, se sustenta en los ahorros de las personas que impulsan el proyecto, y no en los fondos que puedan provenir de otros sectores externos, lo cual incentiva un problema en materia de ganancias, crecimiento y rentabilidad de la microempresa. Resulta oportuno conocer cómo se manifiesta esta situación en la región de Quevedo, a partir de los datos obtenidos por mediación de la encuesta.

Gráfico 1. Inicio de los negocios del microemprendimiento

ISSN: 1390-9320, Vol. 6, No. 6, diciembre 2019 


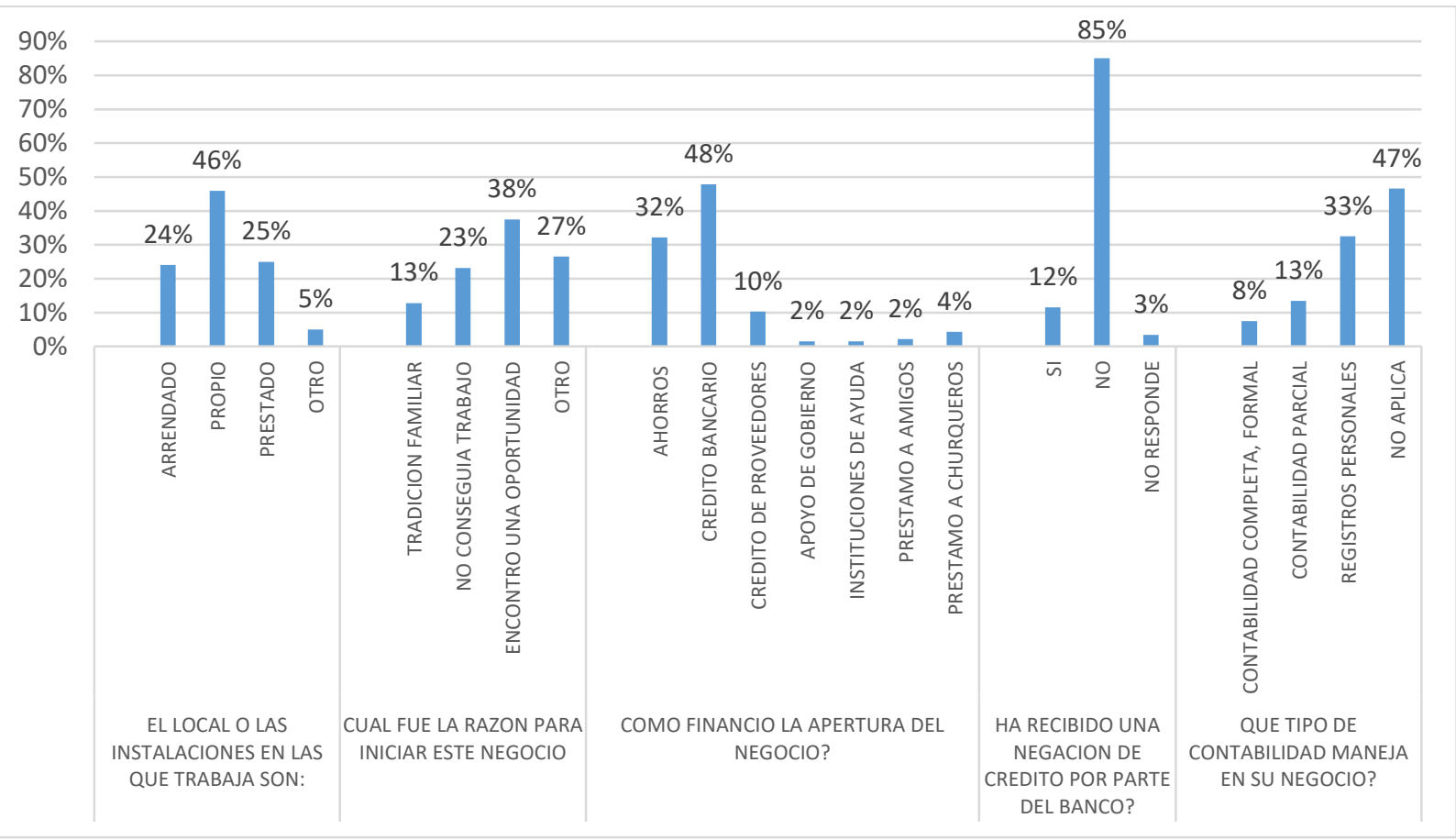

Fuente: Encuesta aplicada a microempresarios Elaboración propia

La apertura de un negocio en el sector del microemprendimiento ha estado generada tradicionalmente por fallas del sistema de empleabilidad con que cuenta el país, que no satisface la demanda de trabajo que existe en la población. Por ello, durante mucho tiempo se pensó a este tipo de negocios como la última opción que tenían las personas para obtener ingresos y subsistir, se definían como puestos laborales provisionales hasta que los individuos encontrasen una mejor opción de empleo, con mejores salarios y sin condiciones de precariedad.

Sin embargo, la realidad en la actualidad muestra otra situación, en la cual las microempresas emergen por el deseo tácito de las personas que ven en este tipo de negocios una oportunidad para desarrollarse. De acuerdo con la encuesta, los microemprendedores no se ven forzados a iniciarse en este tipo de proyectos, sino que eligen esta opción pues les da la posibilidad de actuar con independencia en lugar de suscribirse al trabajo asalariado en otros ámbitos. Resulta una decisión valiente, si se tiene 
en cuenta que los sujetos comienzan este tipo de negocios asumiendo una gran variedad de riesgos, en lugar de sujetarse a puestos laborales ya posicionados y con la garantía de un pago mensual.

En el menor de los casos, las microempresas constituyen lugares vistos como la segunda opción del empleado, que se ve obligado a acudir al sector por no encontrar mejor oferta de empleo. En otros casos, se involucraron porque constituye una tradición familiar, pues en Ecuador predominan dentro del ámbito del microemprendimiento, los familiares, en los cuales generalmente participan los integrantes de la familia o personas muy cercanas a esta. Este tipo de negocio casi siempre es administrado por el cabeza del núcleo familiar, un hombre en la mayoría de las ocasiones, que se desenvuelve cual si fuera un líder y es quien toma las principales decisiones en relación con el negocio.

Ello explica por qué las organizaciones empresariales se desarrollan en la mayoría de los casos en lugares propios o prestados, casi siempre consisten en locales anexos a la vivienda de la familia o que pertenecen a algún miembro de esta, en otros casos de trata de propiedades pertenecientes a quienes ayudaron a la conformación del negocio, ello se ve como un ahorro en materia de renta de locales. En este tipo de lugares tanto empleados como empleadores se sienten más comprometidos con el cuidado del lugar, con mayor sentido de pertenencia.

En otros casos se trata de locales arrendados, lo que supone devengar una cifra de las ganancias por este concepto y por asuntos de mantenimiento y reparación. Este constituye otro de los gastos que debe tener en cuenta la administración de la microempresa para la repartición de los presupuestos y las ganancias con las cuales se cuenta.

Por otra parte, como se puede observar en los resultados de la encuesta, la mayoría de los microempresarios solicitaron financiamiento al banco para iniciar el negocio, lo cual demuestra que dentro de la población empresarial de la región se incrementa la confianza hacia esas instituciones financieras, contrario a lo que sucedía hace alrededor de 10 años, cuando un estudio reveló que la mayoría de microempresarios se autofinanciaban la iniciación de sus negocios (USAID-Ecuador, 2005), gracias a los ahorros, a regalos de otros familiares y amigos, o a través de préstamos informales.

ISSN: 1390-9320, Vol. 6, No. 6, diciembre 2019 
No obstante, existe un gran número de personas que aún se apoyan más en los recursos propios para realizar la primera inversión del negocio, esto puede tener explicación por el desconocimiento acerca de las fuentes de financiamiento que existen, así como de las ventajas que implica concebir inversiones externas para comenzar un proyecto de microemprendimiento. A ello se suma que habitualmente los microempresarios no solicitan préstamos a las instituciones del sector formal para financiar las necesidades personales o comerciales que van surgiendo en el camino, acceden más a los recursos personales y del sector informal, lo cual en gran medida es la razón del bajo crecimiento en ganancias y los inferiores índices de rentabilidad.

Otras fuentes de financiamiento inicial menos demandadas lo constituyen los proveedores, así como los prestamistas informales (conocidos en el argot popular como el acceso a chulqueros y concebidos como un delito); también se recurre en menor medida a los préstamos de amigos y a instituciones de ayuda. Sin embargo, ese porcentaje al ser tan reducido, no resulta significativo para tener en cuenta en la investigación.

Estos resultados son posibles gracias a que los bancos se posicionan como fuertes colaboradores de los negocios de microemprendimiento, en tanto, la gran mayoría de los encuestados refirieron que su solicitud de financiamiento no había sido rechazada por la institución bancaria. Ello demuestra que existe una mayor cultura respecto a la influencia que ejercen las microempresas dentro de la economía de las localidades y una mayor confianza hacia sus posibilidades de éxito, de manera que se generan redes de apoyo dentro de la infraestructura financiera del mercado en los cuales los bancos juegan un papel destacado. Por ello, solo una ínfima parte de la población consultada refiere que su solicitud había sido rechazada.

Ahora bien, independientemente de las fuentes de financiamiento que sean empleados por el microempresario, la rentabilidad y obtención de ganancias por parte del negocio responde al tipo de contabilidad que emplean para realizar un uso adecuado de los recursos y medios de producción. La contabilidad se encarga de registrar, clasificar y resumir las operaciones económicas que desarrolla la empresa, con el objeto de tener acceso a datos de carácter financiero que favorezcan la posterior toma de decisiones.

ISSN: 1390-9320, Vol. 6, No. 6, diciembre 2019 
Sin embargo, resulta alarmante cómo la mayoría de los encuestados refirieron no hacer uso de la contabilidad para gestionar su empresa, y otra cifra mayoritaria se limita a la realización de registros personales, basándose solamente en la experiencia y el sentido común. Otros solamente realizan una contabilidad parcial y una muy inferior cifra de personas se apoya en la contabilidad completa y formal.

Desconocer cuáles son los avances obtenidos en determinados periodos, así como desatender el control de las operaciones y transacciones, limita al microempresario a la hora de asumir los riesgos dentro del mercado; asimismo, se amplía su vulnerabilidad ante las inspecciones que regulan el aprovechamiento de las organizaciones empresariales y ante las entidades tributarias. Por otra parte, poder acudir a datos sobre los ingresos, costos y gastos de operaciones posibilitaría contar con antecedentes para la futura proyección financiera y el estudio de impacto del producto o servicio en el mercado habitual o nuevos mercados. Ello eleva la seguridad no solo de los dueños de los negocios, sino que extiende la imagen de confianza de proveedores, propietarios de locales, instituciones financieras.

Gráfico 2. Formalización como contribuyente.

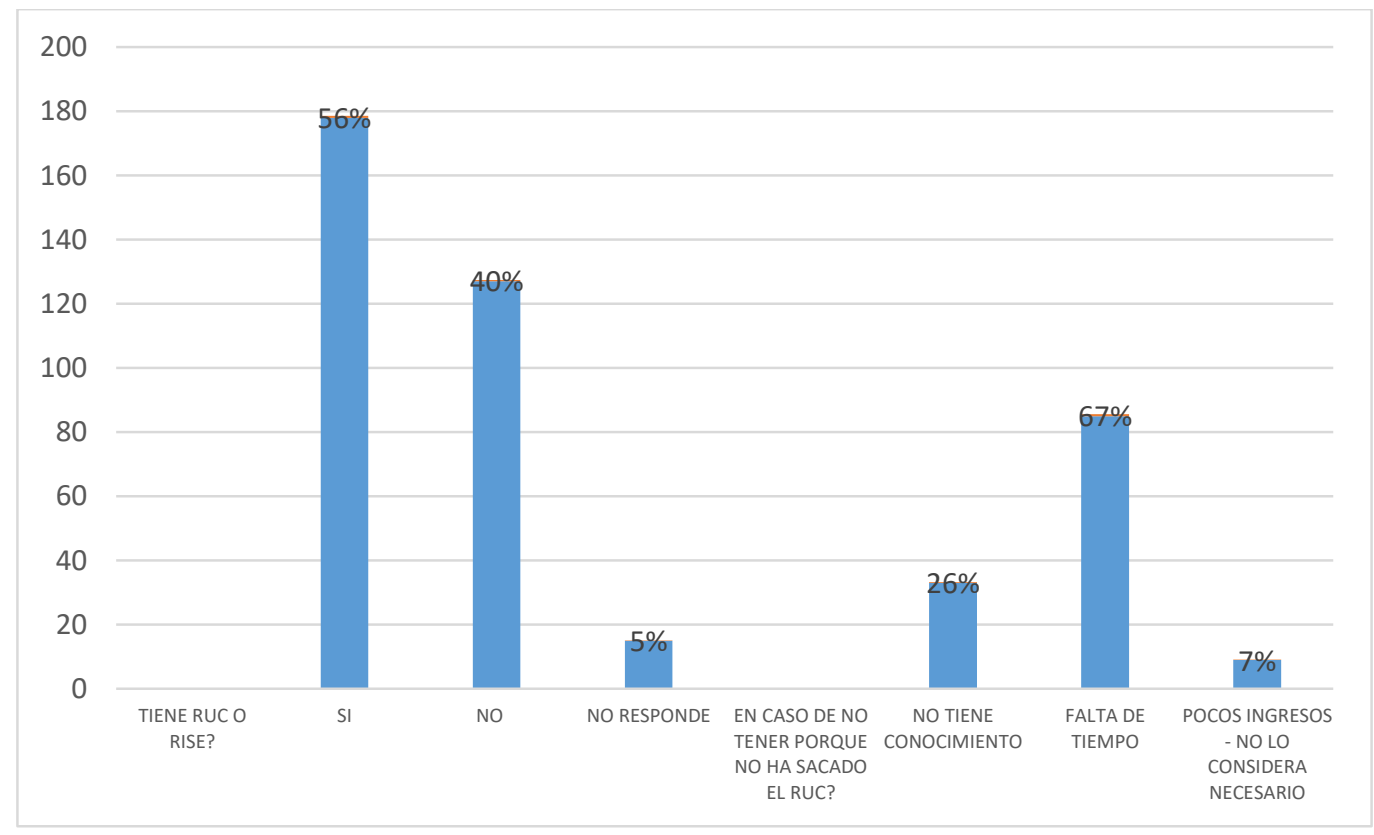

ISSN: 1390-9320, Vol. 6, No. 6, diciembre 2019 
Elaboración propia

Fuente: Encuesta aplicada a microempresarios

Uno de los aspectos que influyen en la imagen de los microemprendedores frente a las instituciones financieras o de cualquier otro orden, es su carácter formal ante las entidades que viabilizan la contribución tributaria. De manera general, los individuos en el país desconocen cuáles son los documentos que deben tramitar para iniciar y permanecer legal en determinada actividad económica. EI RUC o Registro Único de Contribuyentes es el nombre que recibe el registro de contribuyentes en Ecuador, con el cual los microempresarios formalizan su actividad al tiempo que asumen las obligaciones tributarias. Mientras tanto, el RISE, denominado Régimen Impositivo Simplificado ecuatoriano, constituye un nuevo régimen de incorporación voluntaria que reemplaza el pago del IVA y del Impuesto a la Renta a través de cuotas mensuales, pero no todos los empresarios pueden beneficiarse de esta modalidad pues reúne diferentes criterios de selección referidos fundamentalmente a los índices de ingresos anuales, capacidad de empleabilidad, la actividad que ejecuta.

Muchas personas ignoran en qué consiste el RUC o lo confunden con el RISE. Hay que señalar que esta situación se manifiesta entre las personas que fueron consultadas para la presente investigación, pues si bien la mayoría de los microempresarios presentan el RUC, otra importante cifra de ciudadanos carece de esta, entre otras razones, porque no han dedicado tiempo para adquirirlo, otros por desconocimiento de las ventajas de desarrollarse en el ámbito formal, y en el menor de los casos porque no lo consideran necesario debido a sus escasos ingresos.

Gráfico 3. Solicitud de apoyo a instituciones.

ISSN: 1390-9320, Vol. 6, No. 6, diciembre 2019 


\section{Revista Científica ECOCIENCIA}

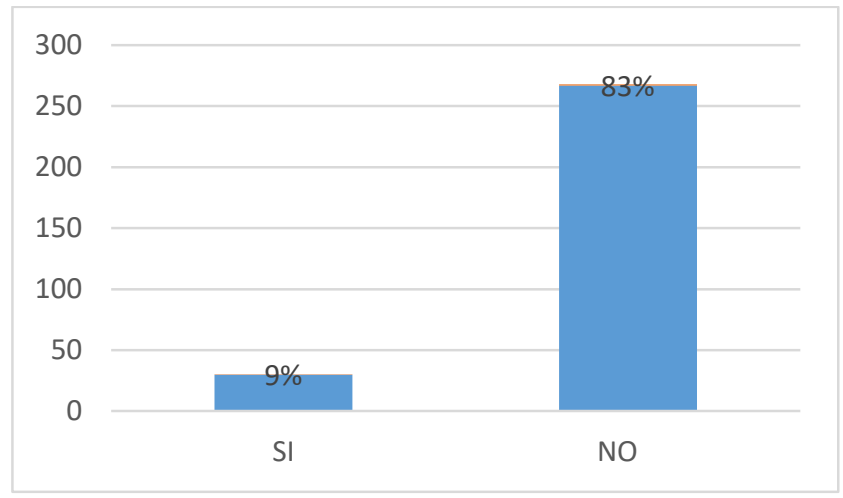

Elaboración propia

Fuente: Encuesta aplicada a microempresarios

Resulta apreciable cómo la gran mayoría de los microempresarios encuestados han estado obligados a requerir algún tipo de apoyo por parte de instituciones privadas y públicas. Una de las razones de esta demanda puede estar asociada a las fallas del financiamiento del negocio. Por lo general, las empresas se ven perjudicadas por la improvisación de sus dueños y administradores, pero el mercado obliga a cumplir con ciertas reglas que implican una mayor rigurosidad y capacitación de los emprendedores.

Entre las necesidades por las cuales generalmente los empresarios solicitan apoyo devenidos créditos se encuentra la adquisición de materias primas; locales y equipamientos con mejores condiciones; capacitación para perfeccionar las destrezas de dirección y de producción, y en el uso de tecnologías sofisticadas. El apoyo puede ser solicitado también debido a una baja demanda y ventas de los productos, que se traduce en una reducida tasa de ingresos, así como el no contar con el total de recursos necesarios para aprovechar las oportunidades de posicionamiento en el mercado y generar crecimiento.

Resulta pertinente establecer un diálogo entre los apuntes teóricos y los datos ofrecidos por la encuesta para explicar porque el financiamiento no debería ser un obstáculo para el desarrollo del microemprendimiento en el país. 


\section{DISCUSIÓN}

El manejo del financiamiento de una empresa determinada resulta uno de los procedimientos más complejos dentro del mundo de los negocios, sobre todo cuando se trata de entidades pertenecientes a los microemprendimientos que, por lo general, se encuentran en situaciones de mayor vulnerabilidad debido a las externalidades del mercado, la dinámica de las grandes empresas, el flujo de inversionistas, el inacceso de los proveedores, la falta de apoyo por parte de la estructura estatal, la escasez de recursos y de personal capacitado en materia de dirección y de producción.

Para Maldonado (2009), el principal incentivo para el éxito financiero de una entidad empresarial radica en el conocimiento que puede presentar el empresario. Al decir del autor, si la persona no domina los aspectos acerca del manejo de la contabilidad y las finanzas es preciso encontrar a un especialista preparado en operaciones que tienen que ver con el monto, plazo de financiamiento necesitado, moneda o reajustabilidad, flujos de amortización, mercados accesibles, nivel de riesgo crediticio, costos transaccionales asociados a cada alternativa.

El diseño financiero debe responder a otras particularidades de la microempresa (Quiñónez, 2013), en aspectos como el sector industrial en el cual participa, el personal contratado, los ingresos y gastos por periodo, el aprovechamiento que pueden tener las inversiones, las mejores fechas del año que constituyen oportunas para tomar riesgos. Sin embargo, según el estudio implementado aún persiste gran ignorancia respecto a las ventajas de mantener un control financiero sobre las actividades de la microempresa, lo cual limita el conocimiento para tomar determinadas decisiones durante el desempeño de las acciones.

En ese sentido, según señala González (2010), para sortear el ambiente altamente competitivo en el cual opera la microempresa, es necesario tener una estrategia financiera que ayuden a enfrentar los rigores de las compras de recursos, la baja demanda, el pago de los trabajadores, la proyección publicitaria, la actualización del equipamiento, la capacitación de los empleados. Estas actividades por lo general, demandan un crédito inicial o intermedio, que favorezcan conseguir en el menor tiempo una mayor producción y venta (USAID-Ecuador, 2005).

ISSN: 1390-9320, Vol. 6, No. 6, diciembre 2019 
Como demuestra la investigación empírica, en la actualidad existen mejores circunstancias para acceder a fuentes financieras seguras, como son las instituciones bancarias (González, 2010); pues esas entidades muestran una mayor apertura hacia la colaboración con los microempresarios; quienes se ven favorecidos también por la cooperación de centros de ahorro y crédito, organizaciones no gubernamentales. Sin embargo, aún persiste la desprotección por parte de los programas gubernamentales.

Hay que referir que no todas las microempresas logran beneficiarse de los créditos de las instituciones formales (Valenzuela, 2014 ), pues en la mayoría de los casos deben reunir los requisitos de confiabilidad que, por lo general, demandan los centros de microfinanzas, como es estar suscrito dentro del sector formal, mantener el control sobre la contabilidad de la empresa, tener adecuados antecedentes en las relaciones con otras fuentes financieras, demostrar cierta estabilidad de los ingresos y que estos no excedan los gastos. De ahí que muchos empresarios sufren la negación de este tipo de solicitud o la interrupción del apoyo debido a la inconsistencia de sus actividades.

En otros casos, persisten temores entre los emprendedores, asociados al alto costo de los préstamos; la inconsistencia entre la tasa declarada y la tasa efectiva que les hace sentir el fantasma de la estafa; la sistemática presión para hacer los pagos oportunamente; las multas altas debido a pagos morosos; o los procedimientos excesivos y requisitos exagerados para montos de préstamo pequeños. Sin embargo, en algunos casos las personas comienzan a evaluar las ventajas de los créditos, como es extender el rango de servicios y productos para corresponder mejor a las necesidades del cliente (USAIDEcuador, 2005).

De acuerdo con Anguita (2000), el diseño financiero debe tener como paso primero, determinar cuáles son las problemáticas que la empresa requiere solventar mediante el reajuste de la administración económica hacia lo interno, valorando también los procesos externos (Valenzuela, 2014 ). A partir de ahí, se requiere del reajuste y utilización de los créditos sobre la base de una información válida: datos acerca del producto o servicio bancario, de manera que se logre comparar los costos y beneficios de diversas instalaciones; evaluar las tasas de interés que se proponen; leer exhaustivamente los 
contratos y los anexos; evaluar los antecedentes de la entidad de microfinanzas. Esta orientación rigorosa conllevará hacia una adecuada alternativa de financiamiento.

No obstante estas premisas, las estadísticas demuestran que muchos de los proyectos de microemprendimiento que se inician en Ecuador fracasan al poco tiempo de ponerse en práctica (EKOSNEGOCIOS, 2015). El estudio ratifica este criterio si se tiene en cuenta que, de acuerdo a las respuestas de los encuestados, varios de ellos habían experimentado ese tipo de desempeño en otras empresas. Ello demuestra las debilidades que aun demuestra el emprendimiento en el territorio.

En ese sentido, la solución estaría en reconocer que ese sector podría constituir un producto de interés estratégico nacional, cuando de la defensa de la perspectiva de desarrollo humano y social se habla. Los negocios del microemprendimiento se encuentran muy relacionados con las bajas tasas de desempleo en algunas territorialidades; y la salida de la pobreza de un gran número de la población mundial (CEPAL, 2013). Este sector inyecta sostenibilidad al sistema empresarial en el país, que deberá ser apoyado por instituciones del Estado como los centros educativos, centros que implementan programas de inversión o que promueven el desarrollo local.

\section{CONCLUSIONES}

Los negocios del microemprendimiento basan casi todas sus actividades a corto y mediano plazo en la lógica del financiamiento (Schumpeter, 2012), mucho del éxito que se respira en este sector se garantiza gracias al acceso y manejo de los créditos a partir de la solicitud a fuentes de microfinanzas confiables, ya sea de carácter formal o informal. El financiamiento se convierte en una herramienta clave para que los emprendedores puedan impulsar sus proyectos, lo cual también va asociado con el interés de las instituciones financieras para apoyar al sector.

Todavía otra gran parte de la población que se desenvuelve dentro de las microempresas mantiene cierta desconfianza ante las entidades de microfinanzas, o desatienden la importancia del financiamiento para hacer progresar sus negocios, que se manifiesta por el desconocimiento de las principales entidades que otorgan créditos a microempresarios;

ISSN: 1390-9320, Vol. 6, No. 6, diciembre 2019 
porque no tienen un ejercicio de contabilidad; la baja frecuencia con que pueden realizar inversiones o la actualización de los recursos y equipamiento con los cuales operan.

Los prejuicios negativos que se formulan sobre las instituciones financieras y sus productos y servicios, limitan el acceso a este tipo de entidades, lo cual implica el desaprovechamiento de oportunidades del mercado por no contar con los recursos económicos para asumir riesgos.

Se requiere, por un lado, socializar las experiencias positivas acerca de las relaciones entre emprendedores y empresas de microfinanzas; así como una intervención más evidente del Estado para ofrecer un apoyo y seguridad a los microempresarios.

\section{REFERENCIAS BIBLIOGRÁFICAS}

Anguita, C. (2000). Guía de financiamiento para emprendedores. Asociación del emprendedores de Chile.

Borja, L. (2015). Las Microempresas Comerciales Categoría Tributaria rise en la Ciudad de Machala. Universidad Técnica de Machala: Machala.

CEPAL. (2013). Emprendimientos en América Latina. Desde la subsistencia hacia la transformación productiva. Reporte de Economía y Desarrollo. Bogotá.

Domínguez, E. (2005). Propuesta de una alternativa de Financiamiento para el Hotel Playa de Oro Varadero. Matanzas: Universidad de Matanzas .

Drucker, P. (1985). The discipline of innovation. Harvard business review, 80(8), 95-100.

EKOSNEGOCIOS. (2015). Emprendimiento en Ecuador. EKOSNEGOCIOS.

Escobar,H., Mendoza, E., Boza,J., Tachong, L. \& Alcivar, M. (2018). Análisis estructural y dinámica de los emprendimientos en la Provincia de Los Ríos- Zona Norte (Vol. 1). Ciencia y Tecnología .

González, A. (2010). Apuntes de Organización Industrial: Competencia en Oligopolios. Universidad de Chile.

Kirzner, I. (1998). Competencia y Empresarialidad. Madrid: Unión Editorial.

ISSN: 1390-9320, Vol. 6, No. 6, diciembre 2019 
Maldonado, M. (2009). Factors impacting the Success of ERP Implementation in Small and Medium Enterprises: An empirical assessment from Latin America. ESADE Business School.

Mungaray, A., \& Ramírez, M. (2007). Human Capital and Productivity in Microenterprises. Universidad Autónoma de Baja California.

Quiñónez, M. (2013). Estudio de la competitividad de las Pequeñas y Medianas Empresas (Pymes). Comerciales de Esmeraldas, 2(1).

Schumpeter, J. (2012). The Theory of Economic Development, New Brunswick (6 ed.). London: Transaction Publishers.

Torres, M. (2006). Microempresa, pobreza y empleo en América Latina y el Caribe. Una propuesta de trabajo. OEA.

USAID-Ecuador. (2005). Microempresas y microfinanzas en Ecuador . USAID-Ecuador.

Valenzuela, A. ( 2014 ). Microemprendimiento y determinantes de su éxito. Santiago de Chile: Primavera.

ISSN: 1390-9320, Vol. 6, No. 6, diciembre 2019 